\title{
Conducting polymer composites of polypyrrole and polyindene
}

\author{
A. Bozkurt ${ }^{\text {a }}$, U. Akbulut ${ }^{\text {a }}$, L. Toppare ${ }^{\text {b, } *}$ \\ a Department of Chemistry, Middle East Technical University, 06531 Ankara, Turkey \\ ${ }^{\circ}$ Department of Chemistry, Bilkent University, 06533 Ankara, Turkey \\ Received 30 November 1995; accepted 16 May 1996
}

\begin{abstract}
Polypyrrole-polyindene composites were prepared via electrochemical methods. Two different approaches were utilized. In the first, the electro-initiated polymerization of indene on a platinum electrode was achieved at $2.0 \mathrm{~V}$ versus $\mathrm{Ag} / \mathrm{Ag}^{+}$in acetonitrile. Then the polyindenecoated electrode was used for the electrochemical polymerization of pyrrole at $1.0 \mathrm{~V}$ versus $\mathrm{Ag} / \mathrm{Ag}^{+}$. In the second case, electrochemical coating of platinum electrode with polypyrrole at $1.0 \mathrm{~V}$ versus $\mathrm{Ag} / \mathrm{Ag}^{+}$was carried out and indene was polymerized on the conducting polymer at $2.0 \mathrm{~V}$ versus $\mathrm{Ag} / \mathrm{Ag}^{+}$in acetonitrile medium. The characterizations of these composites were done by FT-IR, scanning electron microscopy (SEM) and thermal gravimetric analysis (TGA). Electrical conductivities were evaluated by two-probe and four-probe methods.
\end{abstract}

Keywords: Conducting composites; Polypyrrole; Polyindene; Electrochemical synthesis

\section{Introduction}

In the last decade, there has been an increasing interest in the synthesis and characterization of conducting polymers. Of these, polypyrrole, which can be obtained in its conducting form by electrochemical oxidation of pyrrole [1], has been studied most extensively because of its high electrical conductivity and good environmental stability under ambient conditions. However, it has poor mechanical properties and processibility. Due to this reason, considerable progress has been made in improving its mechanical properties. These improvements have been achieved by a method of blending with conventional polymers.

The most common approach for this involves electrochemical polymerization of pyrrole on an electrode which is already coated with an insulating polymer [2-10]. The basic aim is to obtain homogeneous composites with good mechanical properties, at least to a certain extent. In these studies low percolation thresholds were achieved with the help of hydrogen bonding between host matrix and polypyrrole [10]. Graft polymers were also obtained via the same method $[9,10]$. The graft polymer films showed different behaviors in differential scanning calorimetry (DSC), scanning electron microscopy (SEM) and FT-IR, as compared with the mechanical mixture of the two polymers. Insolubility of the insulating polymer in a suitable solvent suggested that the

\footnotetext{
* Corresponding author.
}

composite may be a graft rather than a mixture of two polymers.

We have tried two methods to prepare conducting polymer blends of polyindene with polypyrrole. According to these methods, polypyrrole and polyindene composites were prepared potentiostatically. In doing so, one of the components was polymerized on a metal clectrode and served as an electrode for the other in a different cell. The electrochemical polymerization of indene on a platinum electrode was discussed in detail in a previous work [11].

\section{Experimental}

Composite films of polypyrrole-polyindene (PPy-PIn) were electrochemically prepared by using two different sets of experiments.

In the first, $1.0 \mathrm{ml}$ of indene monomer was injected into 60 $\mathrm{ml}$ of acetonitrile in which the electrolyte concentration was $0.1 \mathrm{M}$. Then the electrochemical polymerization of indene on platinum electrode was achieved at $2.0 \mathrm{~V}$ versus $\mathrm{Ag}^{0} / \mathrm{Ag}^{+}$ at room temperature. The electrochemical polymerization of pyrrole onto a polyindene-coated electrode at a constant potential of $1.0 \mathrm{~V}$ versus $\mathrm{Ag}^{0} / \mathrm{Ag}^{+}\left(10^{-2} \mathrm{M}\right)$ was carricd out in a new three-compartment cell ( $0.2 \mathrm{ml}$ pyrrole monomer and $0.1 \mathrm{M}$ electrolyte concentration in $60 \mathrm{ml} \mathrm{CH}_{3} \mathrm{CN}$ ).

Indene was washed with $6 \mathrm{M} \mathrm{HCl}$, then with $40 \% \mathrm{NaOH}$, stirred for $12 \mathrm{~h}$, and vacuum distilled at $24^{\circ} \mathrm{C}$ at $5 \mathrm{mmHg}$ pressure prior to use. 

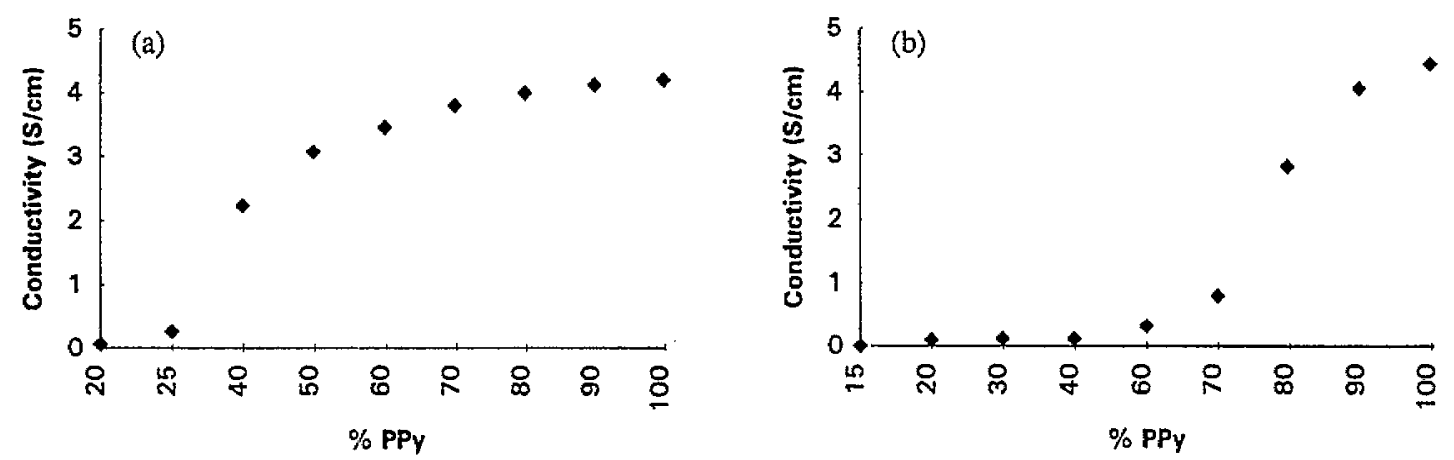

Fig. 1. Electrical conductivity vs. weight percent of PPy: (a) PIn-PPy composite films in the first system; (b) PPy-PIn composite films in the second system.
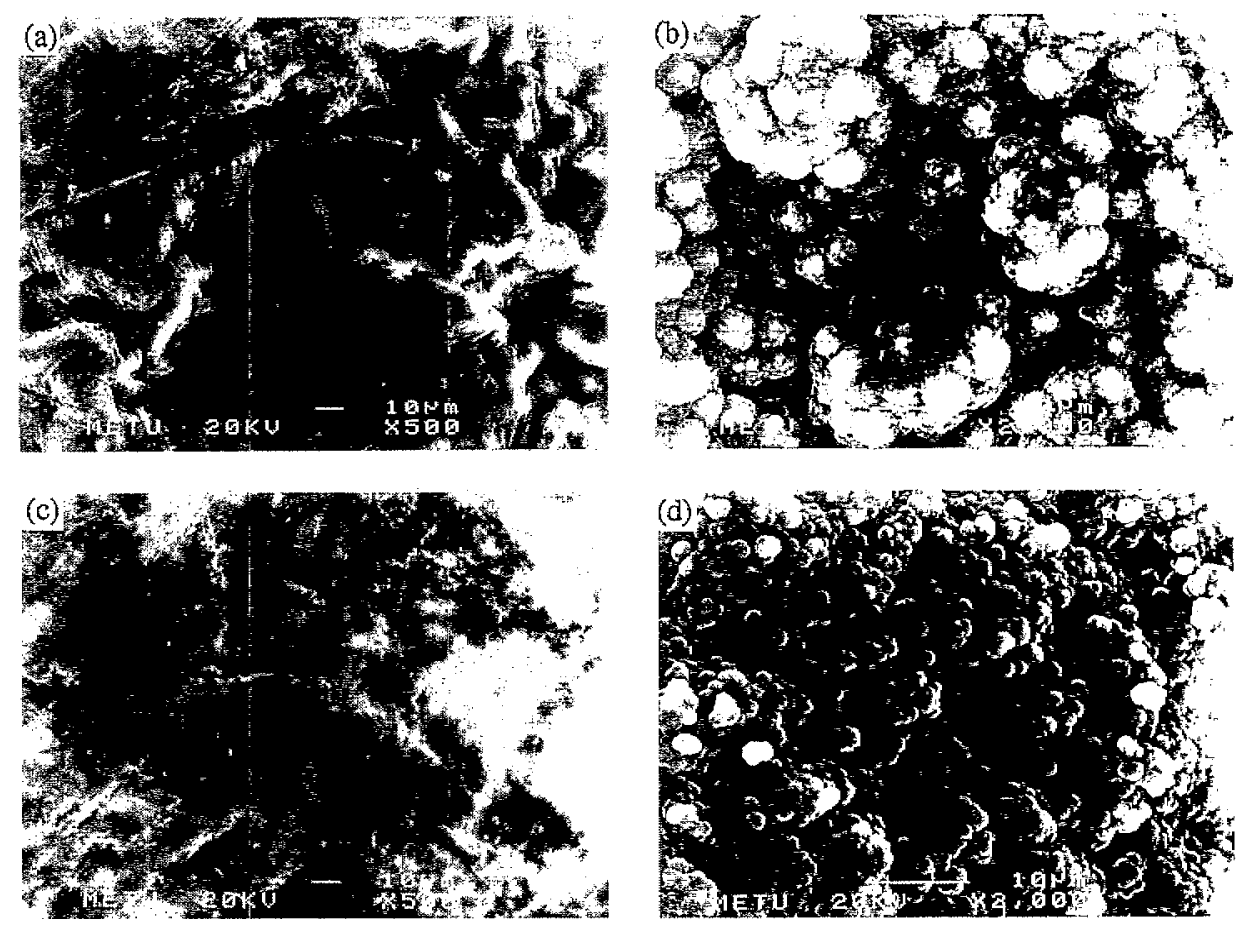

Fig. 2. Surface appearance of SEM films for the first system: (a) unwashed electrode side; (b) unwashed solution side; (c) washed electrode side; (d) washed solution side.
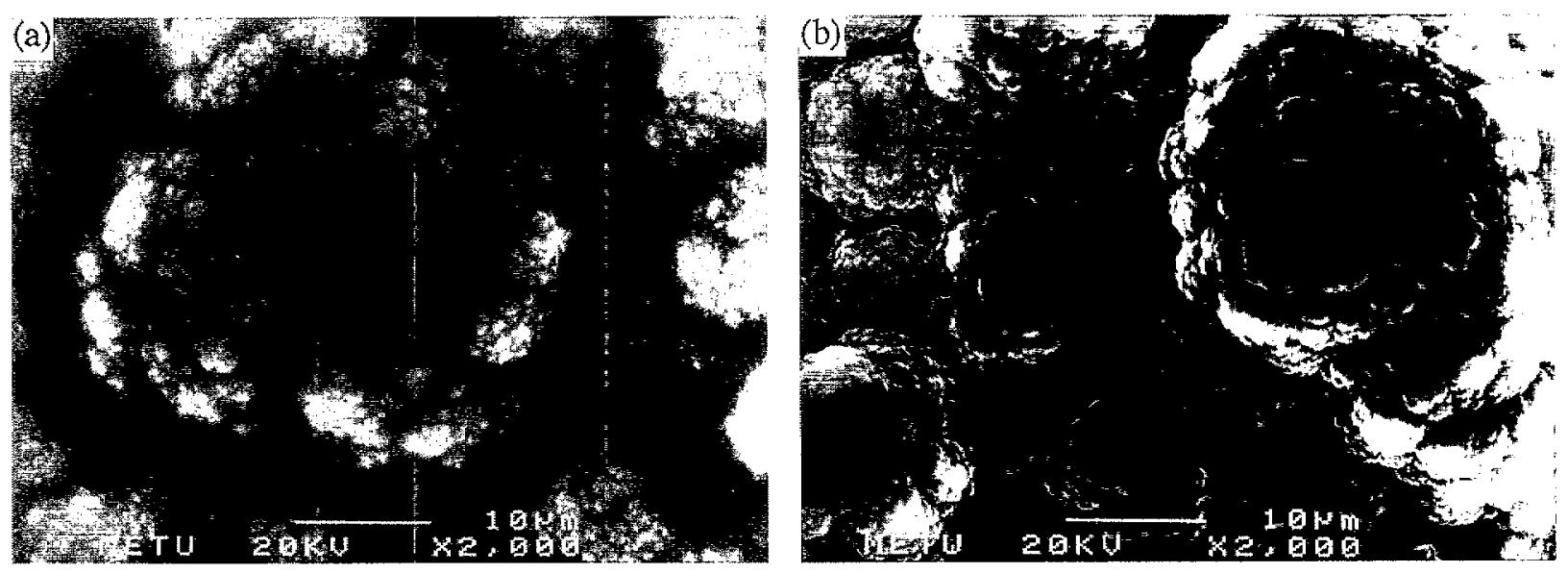

Fig. 3. Surface appearance of SEM films for the second system: (a) unwashed solution side; (b) washed solution side. 

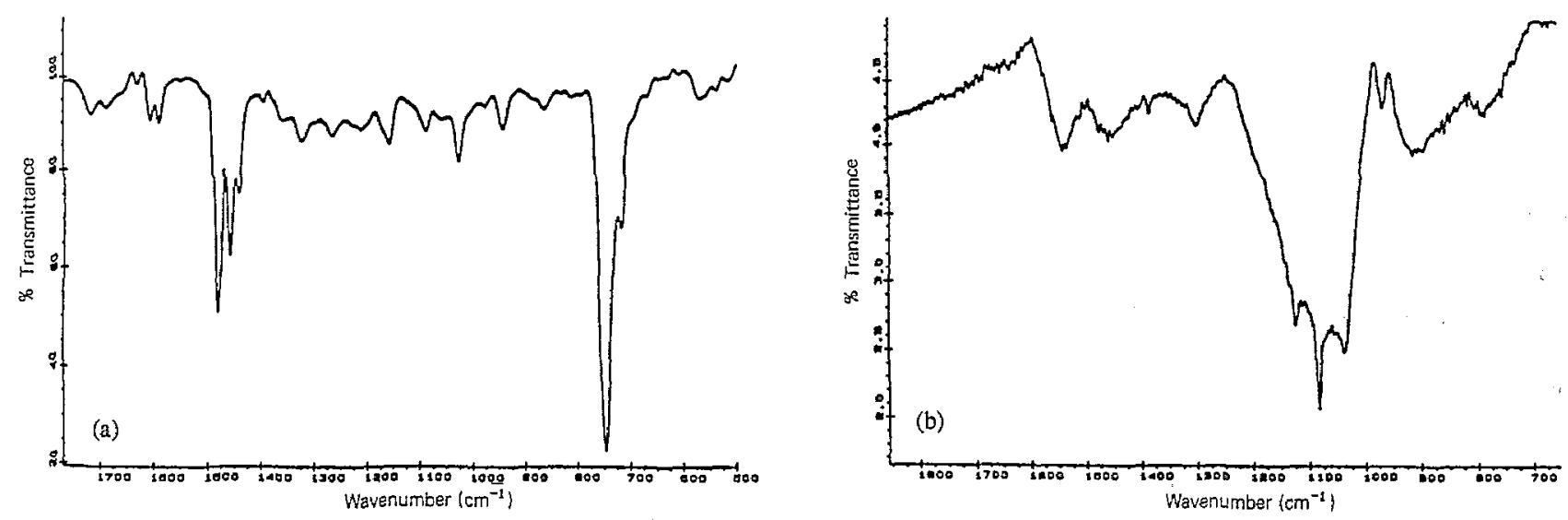

(b)

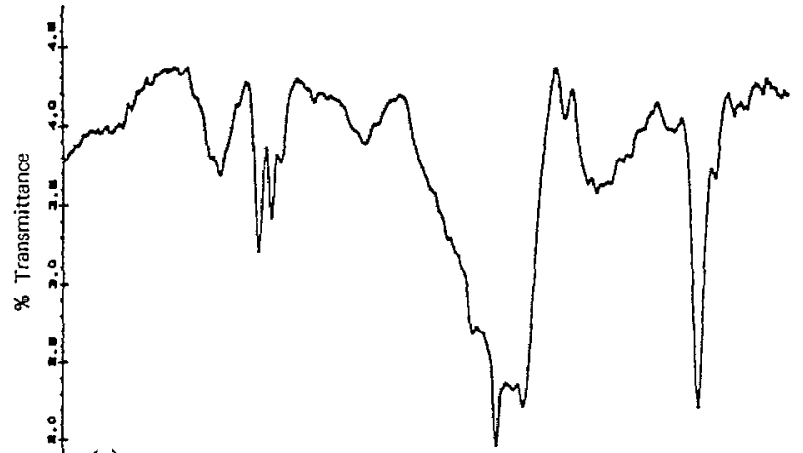

(c)

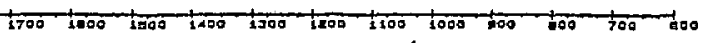

Wavenumber $\left(\mathrm{cm}^{-1}\right)$

Fig. 4. FT-IR spectra of electrochemically polymerized (a) polyindene, (b) polypyrrole and (c) mechanical mixture of the two polymers.
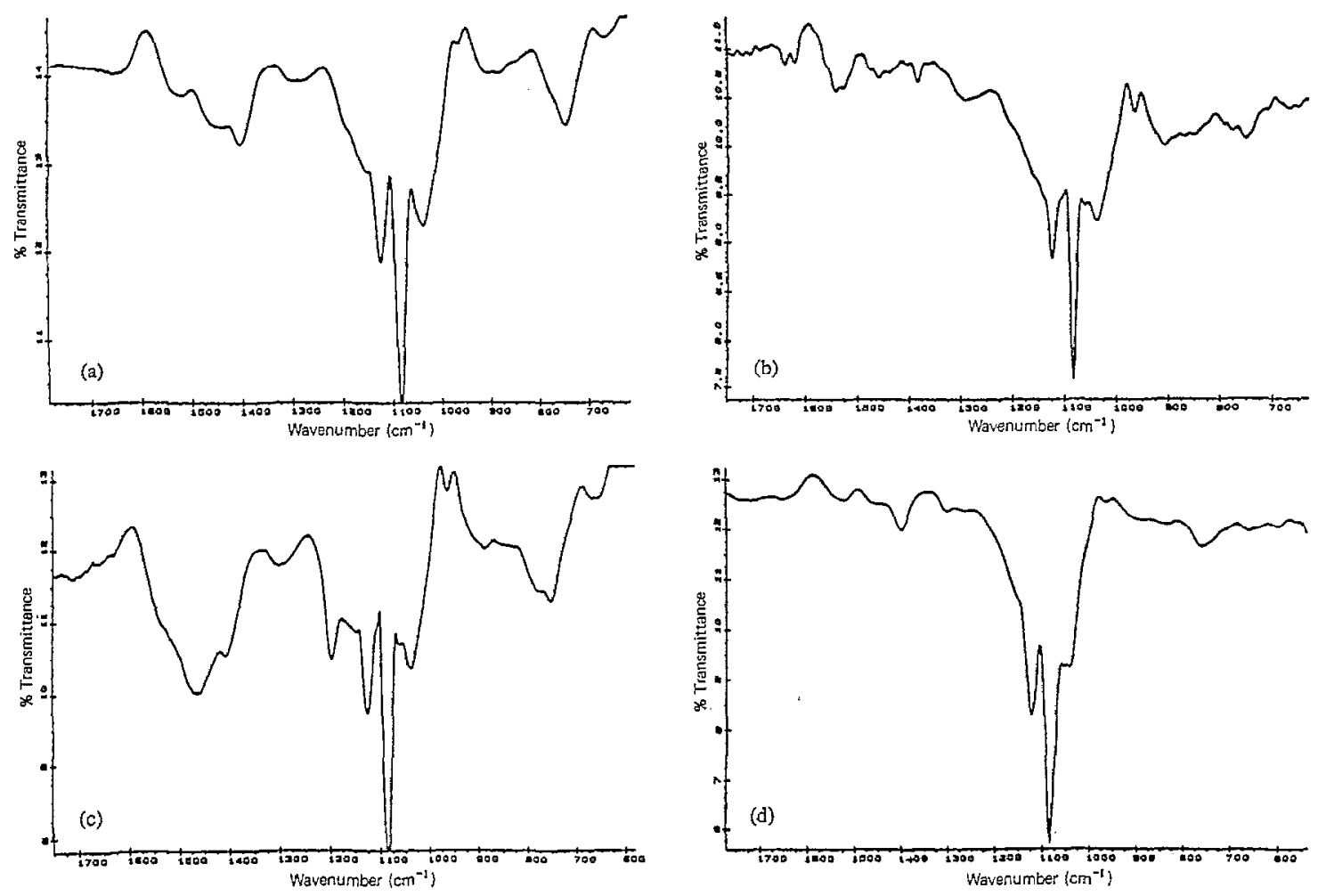

Fig. 5. FT-IR spectra of (a) unwashed electrolytic film of the first system, (b) washed electrolytic film of the first system, (c) unwashcd electrolytic film of the second system, and (d) washed electrolytic film of the second system. 
In the second set the composite films were prepared by the electro-oxidation of indene at a constant potential of $2.0 \mathrm{~V}$ versus $\mathrm{Ag} / \mathrm{Ag}^{+}\left(10^{-2} \mathrm{M}\right)$ in a cell containing $0.5 \mathrm{ml}$ of indene. The electrode here was polypyrrole which was prepared at a constant potential of $1.0 \mathrm{~V}$ versus $\mathrm{Ag}^{0} / \mathrm{Ag}^{+}\left(10^{-2}\right.$ $\mathrm{M}$ ) in a different cell containing $0.2 \mathrm{ml}$ of pyrrole monomer with the same electrolyte concentration.

Potentiostatic polymerizations were carried out in a threecompartment cell equipped with platinum foils $\left(1.5 \mathrm{~cm}^{2}\right)$ as the working and the counter electrodes. The solvent-electrolyte was acetonitrile-tetrabutylammonium tetrafluoroborate (TBAFB). In the first set of experiments blank runs were also carried out to ensure that there were no changes in the weight of the indene-coated electrode. The coatings were also analyzed via FT-IR to see whether there were chemical changes in the polyindene. This is necessary in order to ensure that polyindene itself is electro-inert at the polymerization potential of pyrrole.

Electrical conductivities of samples were measured by the four-probe technique. The composites were characterized by FT-IR (Nicolet 510) and SEM (Cambridge stereo-scan S410). Thermal gravimetric analysis (TGA) was recorded on a Du Pont 2100 instrument. Conductivity temperature relations were measured in a constant-ternperature bath via a twoprobe technique.

\section{Results and discussion}

For both the first and second sets of experiments electrochemical polymerization of pyrrole yielded black films which could be peeled off the electrode surface. It was observed that the solution sides and the electrode sides of the films have the same conductivities. This gives an idea about the homogeneity of the films, at least in terrns of conductivity. It is interesting to note that in the first system (PIn first, PPy later) the conductivities are not greatly affected by the percolation composition, after 70\% PPy content (Fig. 1(a)). However, in the second system (PPy first, PIn second), the conductivities of the films are low until 50\% PPy content has been reached (Fig. 1(b)).

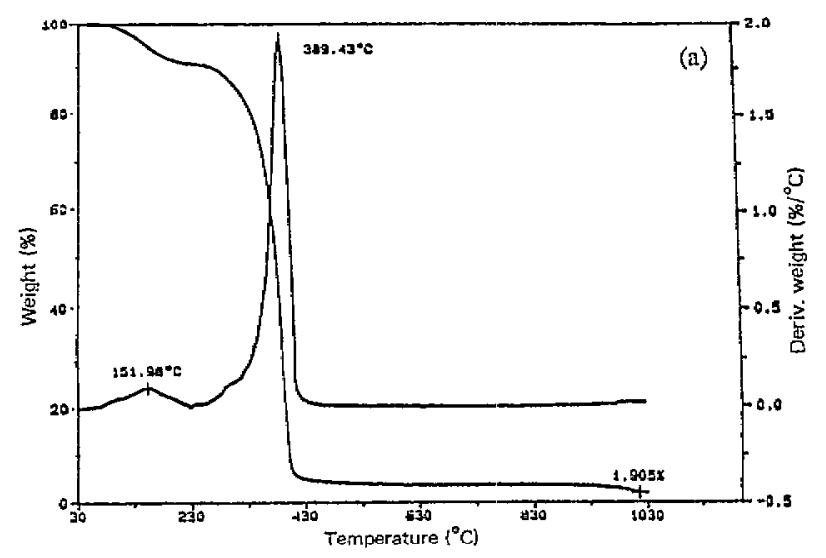

Both types of films were washed for several weeks with chloroform which is a good solvent for PIn. The results show that in the first system there was a $10-20 \%$ loss of PIn by weight, with almost no change in the conductivity observed. On the other hand, in the second system, all the PIn was dissolved in $\mathrm{CHCl}_{3}$ and there are also no marked changes in the conductivity. It seems that the first product is a 1:1 complex of the two polymers with 10-20\% pure PIn in the matrix, whereas the second is a mixture of the two polymers, in which PIn is free to be dissolved. The surface appearances of the washed and unwashed SEM films for both systems are shown in Figs. 2 and 3. When we compare the washed and unwashed films for the first system, there are some changes in the solution side but almost no change at the electrode side. This behavior was attributed to an interaction between the two polymers [8,9]. However, in the second set, PIn was lost completely which shows that the interaction between the two polymers in this case is just a physical adhesion.

FT-IR spectra of pure polymers and mechanical mixtures show different features compared with the electrolytic films (Fig. 4). The spectrum of PPy yields an intense band at 1083 $\mathrm{cm}^{-1}$ coming from the dopant $\left(\mathrm{BF}_{4}{ }^{-}\right)$and has bands at 1161 , 1416,1463 and $1551 \mathrm{~cm}^{-1}$ due to $\mathrm{C}-\mathrm{N}$ and $\mathrm{C}-\mathrm{C}$ stretchings. The N-H wagging band between 909 and $666 \mathrm{~cm}^{-1}$ is also clearly seen. Electrochemically polymerized PIn has characteristic intense bands at $747 \mathrm{~cm}^{-1}$ due to $\mathrm{C}-\mathrm{H}$ out-of-plane bending and at $1478 \mathrm{~cm}^{-1}$ due to $\mathrm{C}-\mathrm{C}$ ring stretching. The mechanical mixture shows all the bands of PIn and PPy; however, in the electrolytic films of both types broadening of the bands is observed. When we look at the washed electrolytic films it is seen that the spectrum of the second system is the same as the spectrum of PPy, but for the first system the characteristic bands that belong to both polymers do not completely disappear, which may be due to a chemical interaction between the two polymers (Fig. 5).

TGA of potentiostatically obtained PIn film reveals a weight loss of about $80 \%$ at around $380^{\circ} \mathrm{C}$ (Fig. 6 (a)). The mechanical mixture of PIn and PPy yields somewhat different patterns due to the presence of PPy. In Fig. 7(a), PPy deposition on a PIn electrode is given, whereas Fig. 7(b) shows

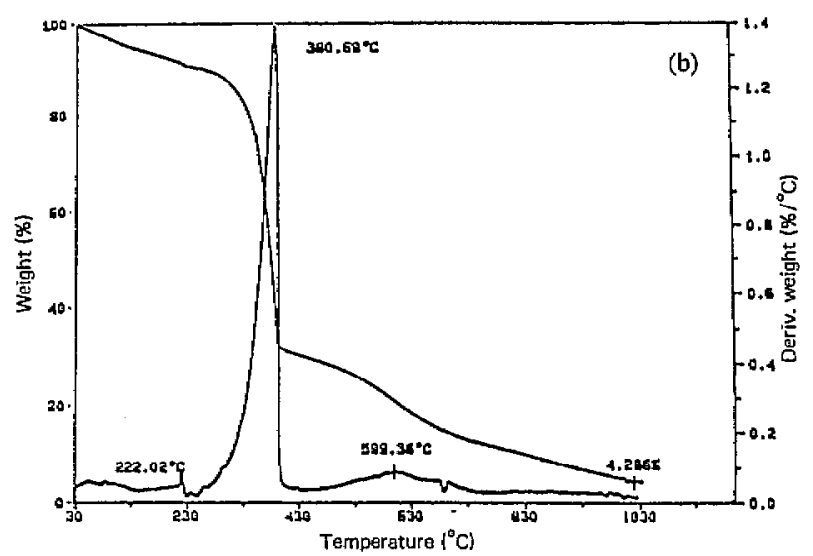

Fig. 6. TGA of (a) PIn and (b) PIn-PPy mechanical mixture (50/50 wt.\%). 

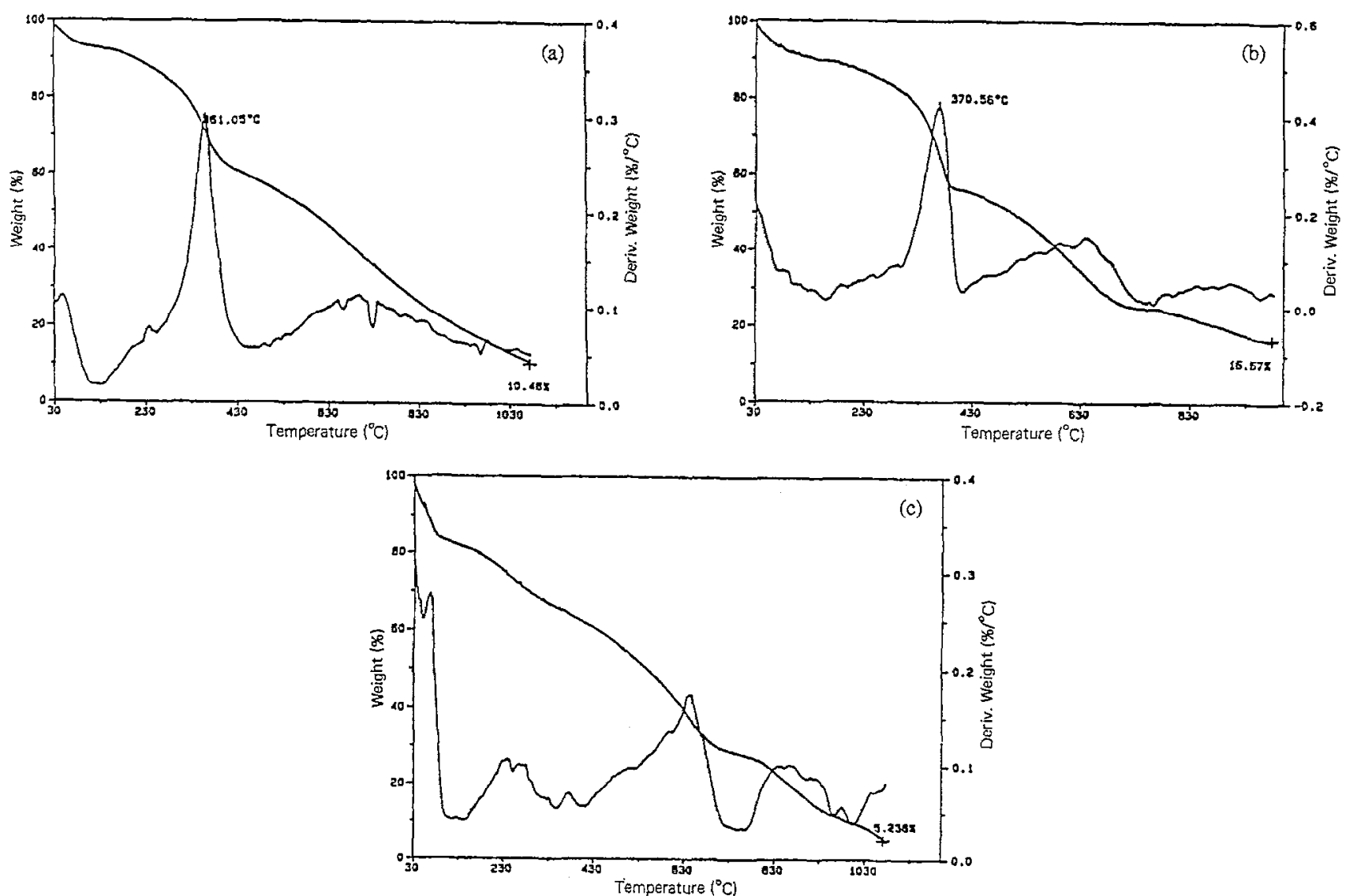

Fig. 7. TGA of electrolytic film: (a) PPy deposition on PIn (50/50 wt.\%); (b) PIn deposition on PPy (50/50 wt.\%); (c) film in (b) washed with chloroform.
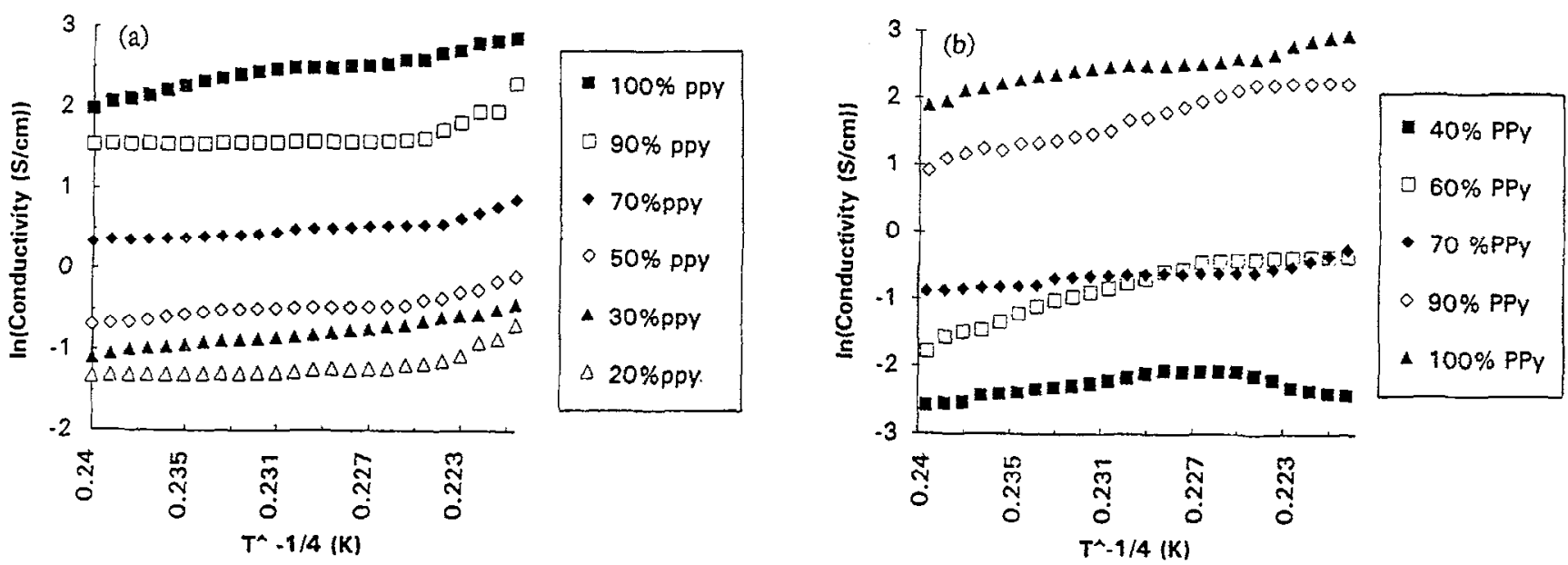

Fig. 8. Temperature dependence of electrical conductivity as a function of percent composition of PPy: (a) first system; (b) second system.

PIn synthesis on a PPy electrode. It is clearly seen from Fig. 7 (c) that no PIn remains in the matrix after washing.

In Fig. 8, temperature dependence of the conductivity of the electrolytic films is presented. Fig. 8(a) shows PIn-PPy copolymer which has a $T^{-1 / 4}$ dependence regardless of the PPy content, whereas for PPy-PIn composite with compositions below $50 \%$ PPy content the conductivity first increases up to around $370 \mathrm{~K}$, then the reverse behavior is observed, i.e., it decreases with increasing temperature. For several heating and respective cooling cycles, the PIn-PPy system ( $30 \%$ in PPy) has a constant $T$ dependence after this first heating (curing) (Fig. 9(a)). However, PPy-PIn composite (40\% in PPy) (Fig. 9(b)) reveals a strange behavior in several heating and cooling runs. The conductivity keeps decreasing with such runs. It seems that the first material, i.e. PIn-PPy, retains the properties of a conducting polymer, yet the second set of products (PPy-PIn) is a simple mixture of the two polymers. 

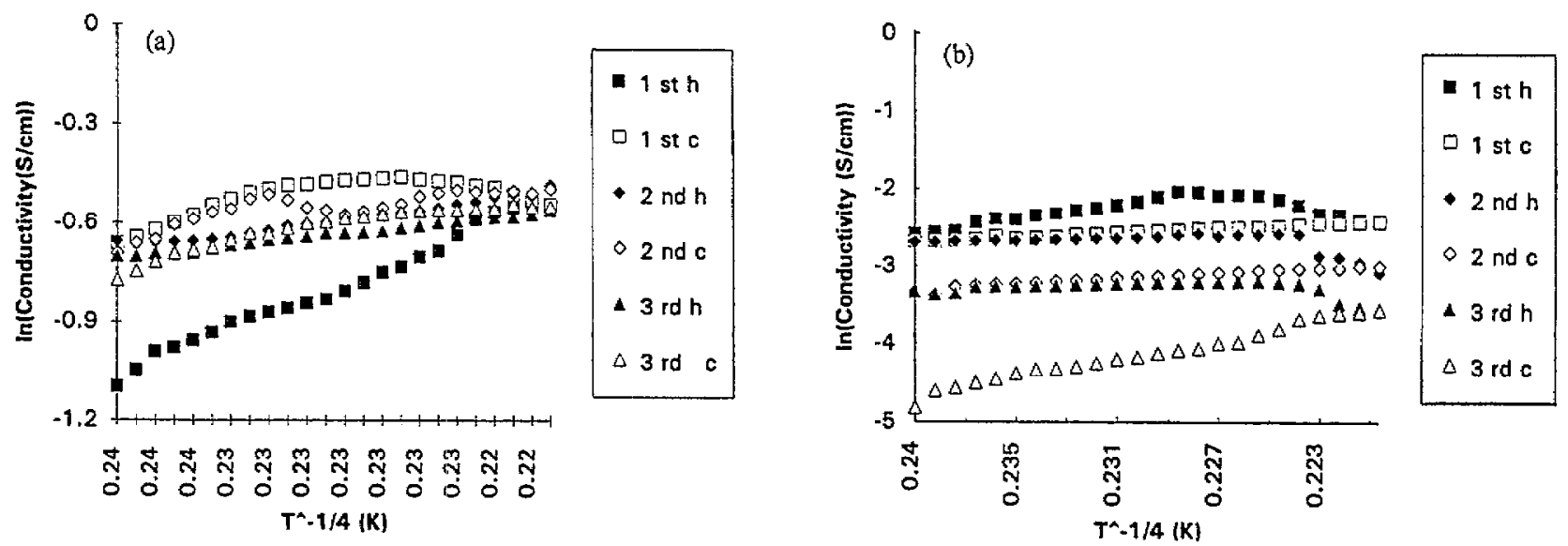

Fig. 9. Heating/cooling curves of (a) first system and (b) second system,

\section{Conclusions}

As the characterization methods reveal, in the first system, PPy-PIn polymer alloy film is a copolymer of the two elements to a certain extent. The second system yields homogeneous composites of PPy and PIn. Nevertheless, this approach shows that PPy can be used as a suitable electrode in the polymerization of indene.

\section{Acknowledgements}

This work is supported by Grants DPT-95, K120498 and TBAG-1422.

\section{References}

[1] A.F. Diaz, K. Kanazawa and G.P. Gardini, J. Chem. Soc., Chem. Commun., (1979) 635.

[2] O. Niwa and T. Tamamura, J. Chem. Soc., Chem. Commun., (1984) 817.

[3] B. Zinger and D. Kijel, Synth. Met, 41-43 (1991) 1013.

[4] G.P. Zhang and X. Bi, Synth. Met., 41-43 (1991) 251.

[5] B. Tieke and W. Gabriel, Polymer, 31 (1990) 20.

[6] Y. Chen, R. Qian, G Li and Y. Li, Polym. Commun., 32 (1991) 189.

[7] X. Bi and Q. Pie, Synth. Met., 22 (1987) 145.

[8] S. Dogan, U. Akbulut and L. Toppare, Synth. Met., 53 (1992) 29.

[9] F. Selampinar, U. Akbulut, T. Yalcin, S. Suzer and L. Toppare, Synth. Met., 62 (1994) 201.

[10] H.L. Wang, L. Toppare and J.E. Fernandez, Macromolecules, 23 (1990) 1053

[11] U. Akbulut, S. Eren and L. Toppare, J. Macromol. Sci. Chem., A21 (1984) 335 . 